\title{
CARS (coherent anti-Stokes Raman spectroscopy) diagnostics of discharges
}

\author{
J. P. Taran \\ Office National d'Etudes et de Recherches Aerospatiales, BP 72, 92322, \\ Chatillon Cedex, France
}

\begin{abstract}
Various types of discharges have been studied recently at ONERA using CARS. They are presented in this communication, following a brief review of the limiting mechanisms.
\end{abstract}

\section{INTRODUCTION}

Most of the applications of Coherent anti-stokes Raman Spectroscopy (CARS) in the area of analytical chemistry have been related to combustion diagnostics. Combustion studies, however, present many severe problems, especially when studying large scale turbulent reactors: the beams are bent and defocused by the index gradients leading to substantial intensity (hence concentration) measurement errors. Further, it is a fact that the modelling of turbulence remains crude and that the comparison between experiments and results of numerical simulations does not yet permit the most fruitful exchange between spectroscopist and modeller.

Recently, a few measurements of photochemical products have been undertaken (ref. 1-5), showing the potential for detection of species at low pressure with the difficult challenge of completing the measurement in precise synchronism with the photolysis pulse. In this area, the exchange between the experimenter and the theorist is far more rewarding. The measurements can be made quantitative. Yet, in our opinion, discharges are an even better field for CARS.

The measurement of quantum state populations in discharges is a difficult problem for the plasma physicist. Emission spectroscopy can be used, but it gives information only about excited electronic states, which are usually far less populated than the ground state. The latter will be detected in absorption, but one does not always find a convenient light source for it. Fluorescence, which is quite sensitive, can also be used. But many of the common species like $\mathrm{N}_{2}, \mathrm{O}_{2}, \mathrm{H}_{2}$, absorb in the far UV, where laser sources are not very efficient. Furthermore, the discharge populates the very same states which are accessed by the laser pump, so that light is emitted spontaneously at the same wavelength as the fluorescence signal, causing a potentially harmful interference. Thus, very little appears to be known today about ground state molecular species, even in conventional discharges such as the glow discharge. Surprisingly, very few papers have been published so far on CARS studies of such plasmas (ref. 6-10), although the initial demonstration of feasibility is quite old (ref. 6). Yet, the application of CARS is here particularly attractive, with its high detection sensitivity, sharp, Doppler-broadened lines, and weak non resonant background.

\section{CARS DETECTION SENSITIVITY IN LOW PRESSURE GASES}

\section{Instrumental particulars}

The CARS instrument used for the experiments reported in this presentation has been described previously. The technical details and experimental procedures are found in (ref. 5, 11, 12). The principal characteristics are the following:

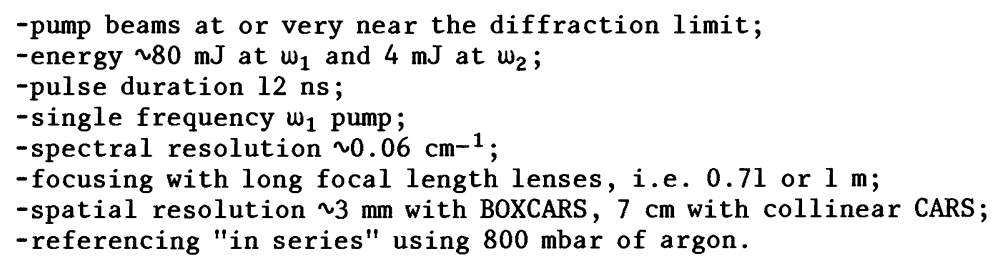




\section{Discussion}

The use of CARS for low pressure gases requires optimal instrumental sensitivity for the detection of metastable states. This is no trivial matter. The natural recourse is to increase the pump laser powers, but this is limited by the onset of vibrational saturation and Stark broadening (ref. 12, p. 35). Both phenomena may become quite pronounced using state of the art CARS set-ups, i.e. with $50-100 \mathrm{~mJ}$ in a doubled Yag laser and $5 \mathrm{~mJ}$ in a narrow band dye laser. To some extent, these phenomena can be tolerated if the instrumental response can be calibrated on a known sample, at about the same translational temperature, and using the same laser powers. A special case, addressed below, is that of the detection of high-lying vibrational states, for which no prior calibration is possible.

Reduction of the interference from these higher-order non-linearities is possible, albeit to a limited extent. Single mode $\omega_{1}$ pumps are preferable. Use of long focal length lenses and of small diameter beams also minimizes the power density in the focal region. However, the beam diameters cannot be reduced much below $5-6 \mathrm{~mm}$ to avoid damage to the dielectrically coated windows. The windows must be mounted close to the lenses. It is then sensible to use focal lengths of $1 \mathrm{~m}$ or below, so as to keep the discharge cells from being prohibitively long and also to preserve some spatial resolution in collinear CARS.

Collinear CARS gives the best detection sensitivity, potentially a factor of 10 better than BOXCARS. However, some non-phase matched nonresonant signal is generated in the windows. The most severe problem is posed by the entrance window. Absorbing filters (eg Schott OG 515) give a slight improvement. The best results were obtained with dichroically coated silica (low-pass, coating facing inside), which was found to generate a signal only about $3 \%$ that of uncoated flats. The exit window also is covered with a high-pass dichroic coating facing inside. With this arrangement, the average level of spurious CARS emissions from the optics is about $40 \pm 10$ photoelectrons per shot with an evacuated cell (ref. 11). Interestingly, the fluctuations on that signal were found to be much larger than expected from the simple Poisson statistics. The non-phase-matched character of the interaction is probably responsible for this phenomenon. Note also that the transmission of the signal collection optics is of the order of $10 \%$ and the phototube efficiency $30 \%$, so that the net emission from the cell is in excess of 200 photons, i.e. quite a large flux. Finally, BOXCARS and non-resonant background cancellation, which both suppressed the background completely, were tried. None was able to improve the signal to noise ratio of the fainter Raman lines and the detectivity, because of the larger signal loss. The best detection sensitivity ever obtained was about $10^{11} \mathrm{~cm}^{-3}$ on the $\mathrm{v}=3, \mathrm{~J}=1$ rovibrational state of $\mathrm{H}_{2}$ at a temperature of $550 \mathrm{~K}$ in the multipolar discharge described below.

Three solutions can be implemented to improve these figures: 1) mix the two pump beams on a dichroic in the vacuum of the discharge cell (Fig. la); 2) use several high pass dichroic mirrors immediately after the entrance window to remove its contribution (Fig. 1b); 3) use a multiple pass arrangement to enhance the signal from the gas (Fig. 1c).

The first solution offers the advantage that the pump beams are made collinear only after traversing the optical ports. However, the beam alignment becomes difficult to perform and its long term stability is problematic. In addition, rare gas referencing, which improves the measurement precision and expedites the recording of spectra, becomes technically difficult. No attempt was made by us to use it. The second one was tried, and was found to reduce the average stray light by a small factor ( 3 to 4 ), but not the fluctuations. This, combined with a small loss in signal from the gas, did not yield an improvement in signal to noise ratio. Finally, the multipass scheme was also tried (ref. 11). A gain in the sensitivity was noted, but not as large as the inverse of the number of passes which is expected; further, that gain was frequency-dependent. The blame rests probably on the dephasing incurred by the waves in the dielectric coatings (ref. 11).

In conclusion, it is felt that in this area of weak CARS signal detection, some work remains to be done to understand unphase-matched generation from windows and dielectric coatings, as well as the dephasing properties of the latter.

\section{DISCHARGE STUDIES}

Three homonuclear diatomics have been studied under specific discharge conditions: $-\mathrm{O}_{2}$ in a surfatron microwave cavity; $-\mathrm{N}_{2}$ in a glow discharge; $-\mathrm{H}_{2}$ in a magnetic multicusp. The salient results of these investigations are presented in this chapter.

\section{$\mathrm{O}_{2}$ in surfatron}

Oxygen undergoes substantial dissociation in microwave discharges. Recombination of the atoms leads to the production of $10-15 \%$ of the singlet delta species. On the other hand, the surfatron microwave cavity provides a high power density discharge volume which is nearly uniform (ref. 13). This last property invites the use of the more sensitive CARS 

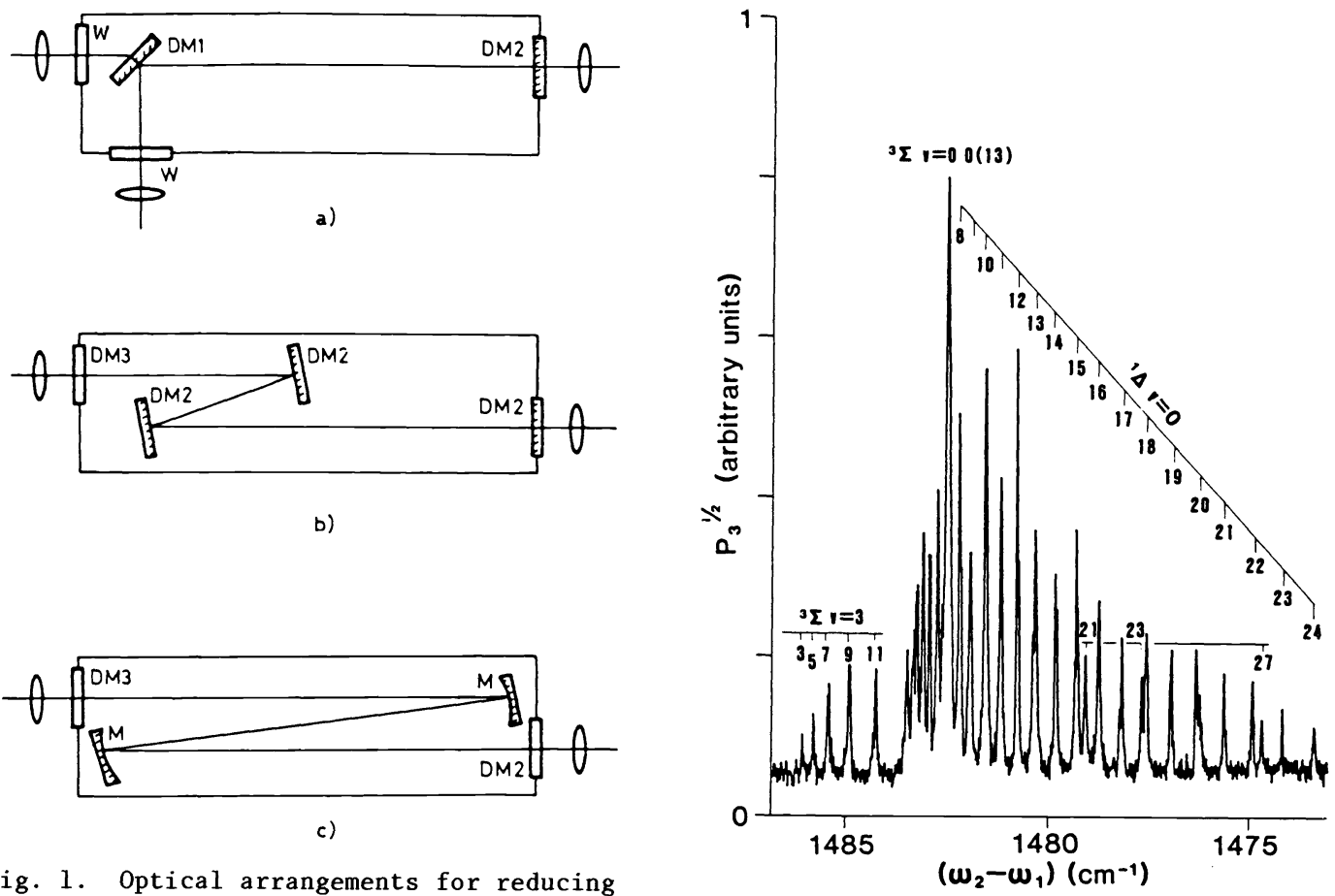

Fig. 1. Optical arrangements for reducing background generated by optics in CARS; W: AR-coated window; $\mathrm{DMl}$ : beam-combining dichroic; DM2: high-pass dichroic; DM3: low-pass dichroic; M: wide band concave mirror.

Fig. 2. Spectrum of singlet delta oxygen in surfatron discharge in $1 \mathrm{~cm}$ diameter tube with $\sim 190 \mathrm{~W}$ at 2.5 mbar pressure. Pure oxygen was flown through the discharge. Collinear CARS was used; all polarizations were parallel.

collinear beam arrangement, and the first spectra were recorded in that mode. The $\mathrm{O}_{2}{ }^{1} \Delta$ was looked for in a $190 \mathrm{~W}$ surfatron discharge. One of the spectra obtained is displayed in Fig. 2. The main Q-branch is visible. The weaker $v=3$-branch and the strong $0(v=0$, $J=13$ ) line of the ground state, ${ }^{3} \Sigma$ oxygen are also seen. The rotational temperature of the singlet state measured from this spectrum is $410 \mathrm{~K}$; at the same location, one measures a rotational temperature of $1100 \mathrm{~K}$ and a vibrational temperature of $1500 \mathrm{~K}$ on the triplet state using BOXCARS. The difference in the rotational temperatures of the singlet and triplet states reveals the bias caused by the inadequate spatial resolution of collinear CARS. Subsequent measurements performed on the singlet state using BOXCARS have shown the concentration to be small in the discharge region and to increase to a maximum several centimeters downstream in the post-discharge zone, while the rotational temperature drops from 800 to $400 \mathrm{~K}$. These tests demonstrate one more time the need to use collinear CARS with caution.

\section{$\mathbf{N}_{\mathbf{2}}$ in glow discharge}

The behavior of nitrogen in discharges has attracted considerable interest since the discovery of the $\mathrm{CO}_{2}$ laser. Many models have been developed, but none had been tested experimentally. Recently, the use of CARS has shown this to be possible (ref. 8, 10). The present report is based on work done in preparation for a study of nitridation (ref. 14). The measurement of rotational temperatures and of the vibrational population distributions was undertaken. Results were obtained in the discharge and in the post-discharge regions.

Research grade $\mathrm{N}_{2}$ was flown through a 2-cm diameter pyrex tube. The discharge length was $40 \mathrm{~cm}$, the current $80 \mathrm{~mA}$. The spectra of Figure 3 are typical. They were recorded at 5 mbar pressure using collinear CARS for all vibrational levels but $v=0-5$. The rotational temperature was $550 \mathrm{~K}$ for all of these levels, with an uncertainty ranging from $\pm 20 \mathrm{~K}$ for the first 10 vibrational levels and degrading to $\pm 100 \mathrm{~K}$ for the highest ones. The vibrational distribution was clearly non-Boltzmann (Fig. 4). Note that CARS only gives population differences, which leaves some uncertainty on the absolute number densities of the high v's. The most reasonable assumption (conservation of the slope between $\mathrm{v}=13$ and 14) was used to project the value of $v=15$. Population calculations were also done assuming slope changes of $\pm 10 \%$ (adjacent lines) to show the most extreme distributions compatible with the instrumental error. The experimental data can be compared with the simple model of Treanor as modified by Gordiets and coworkers (ref. 15). The dotted curve shows the prediction of that analytical model using the measured values of the vibrational and rotational temperatures. The discrepancy appears large at high v; it can be explained 


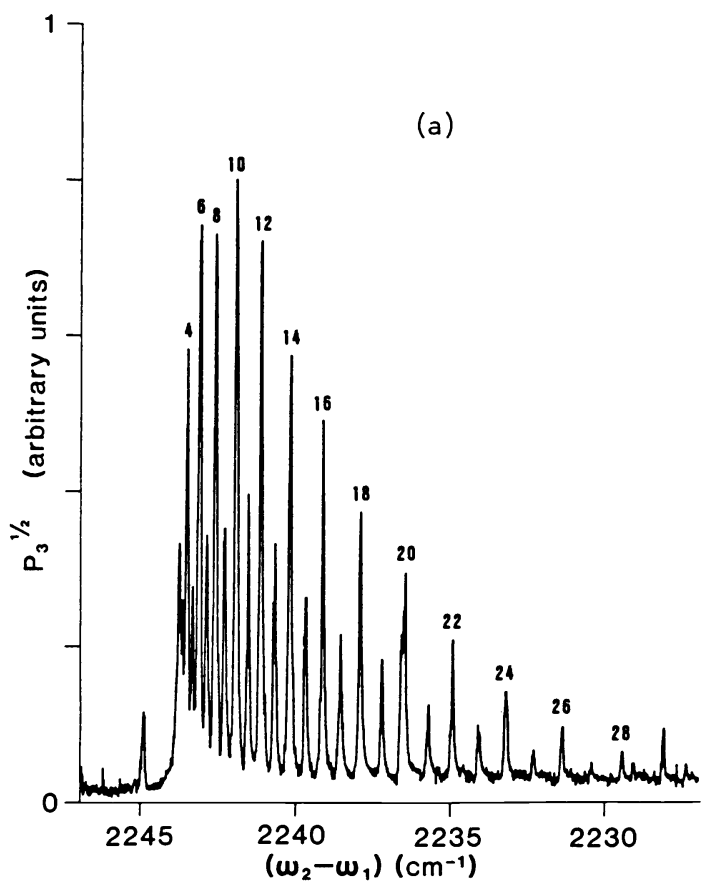

(b)

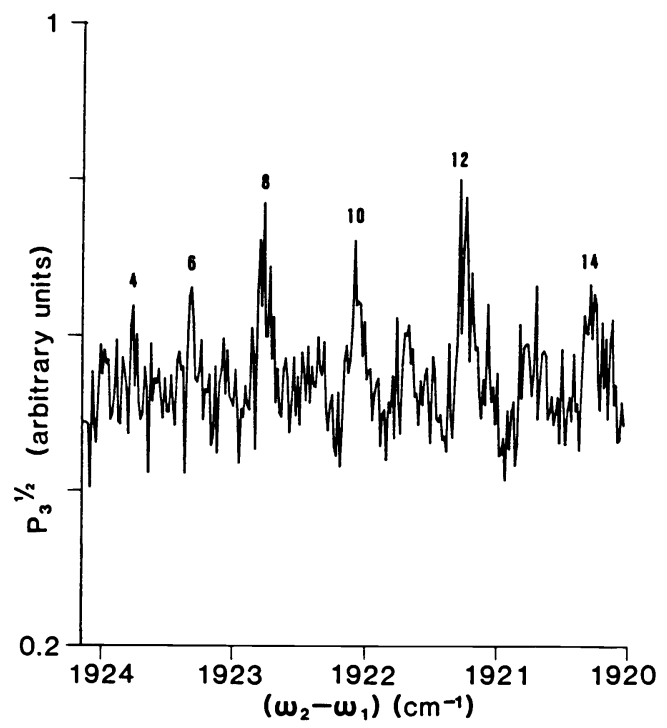

Fig. 3. Spectra of $N_{2}$ in glow discharge at 5 mbar pressure. (a) $v=3$ with BOXCARS; (b) $\mathrm{v}=13$ with collinear CARS; parallel polarizations.

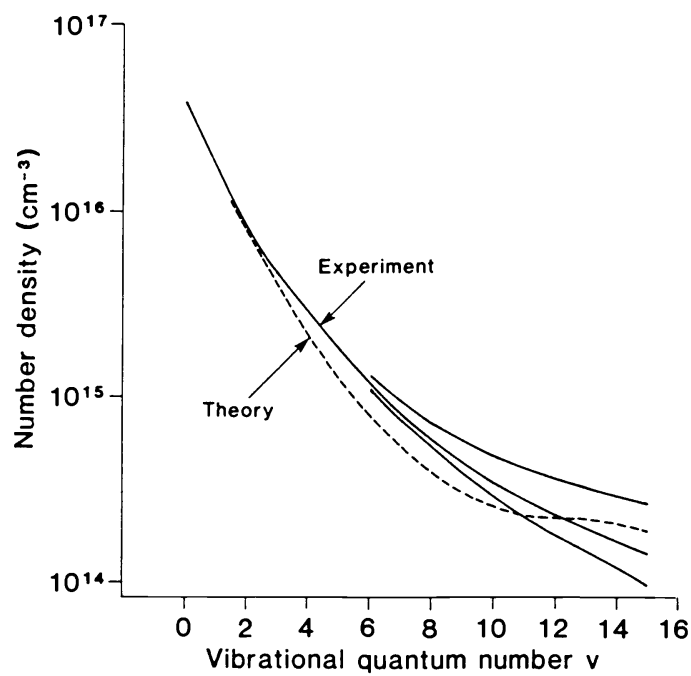

Fig. 4. Vibrational distribution obtained with the conditions of Fig. 3.

by the effect of wall collisions and electronic collisions which are not accounted for in the model. Other models by Capitelli and coworkers (ref. 16) and by Brunet (ref. 17) include electronic effects and yield more accurate predictions (ref. 14).

The major problem that was encountered in these experiments is saturation, which was found to be vibrational-state-dependent. This problem is not severe so long as a calibration can be performed under precisely the same CARS instrumental conditions using a sample at similar temperature and pressure. However, this works well only for the lowest rovibrational states which are thermally populated near room temperature. It is more difficult to prepare known populations in, e.g., high-lying vibrational levels, hence the need to simulate the error caused by saturation on the entire vibrational manifold.

A model of the saturation was developed and good agreement was found with experimental data for the first three vibrational levels using BOXCARS and up to $v=8$ with collinear CARS, for which less saturation is seen, as expected. The data of figure 4 are actually corrected using the results of the model to extrapolate the correction to the higher vibrational levels. Typically, the correction applied to the population difference of $v=$ 4 is $25 \%$ and reaches a factor of 1.9 for $v=14$. Note that the results are also corrected for vibrational anharmonicity (ref. 18). This correction is small (i.e. $20 \%$ on $v=14$ ). These results will be published elsewhere (ref. 19). 


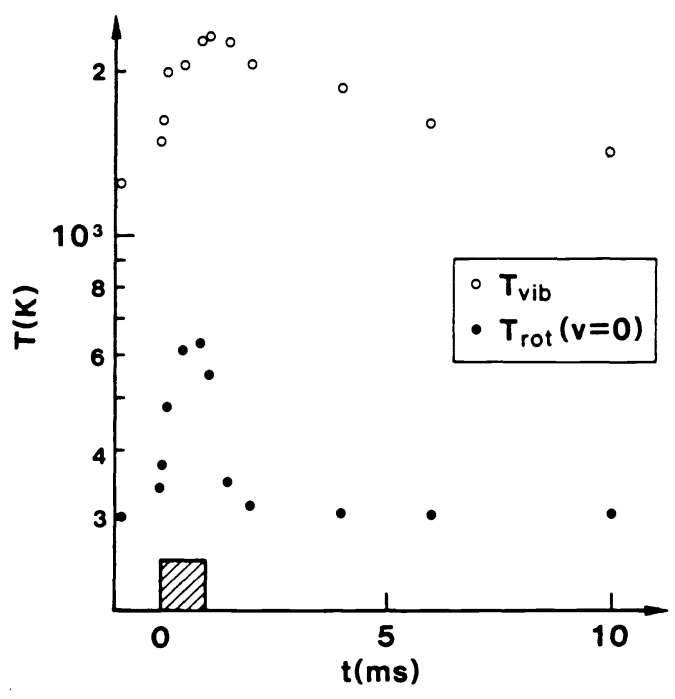

Fig. 5. Response of temperature values to $1 \mathrm{~ms}, 10 \mathrm{~A}$ pulse; $55 \mu \mathrm{bar}$ pressure; notice overshoot of rotational temperature above steady-state value of $550 \mathrm{~K}$ because of adiabatic effects.

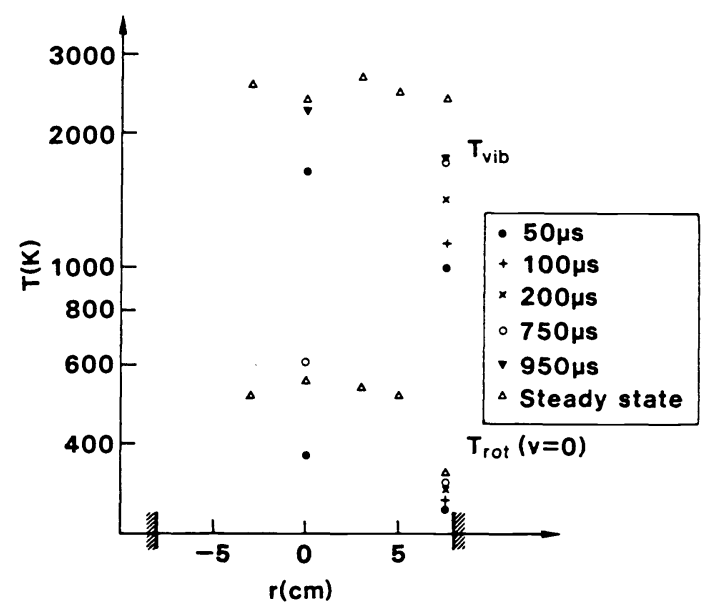

Fig. 6. Radial profiles and temporal response of rotational and vibrational temperatures; same conditions as for Fig. 5; the origin of times is the front edge of the discharge pulse.

\section{$\mathrm{H}_{2}$ in magnetic multipolar discharge}

The analysis of a low-pressure discharge with magnetic multipolar confinement of primary electrons emitted by a tungsten filament has begun several years ago in our laboratory (ref. 9, 11). Measurements were done in a $16 \mathrm{~cm}$-dia., $20 \mathrm{~cm}$-high reactor, at $55 \mu \mathrm{bar}$ pressure, and using a $90 \mathrm{~V}, 10 \mathrm{~A}$ discharge. The main conclusions of these previous studies are the following: 1) the rotational distribution strongly deviates from the Boltzmann law for $\mathrm{J}>5$; the rotational temperature deduced from $\mathrm{J}<5$ is about $530 \mathrm{~K}$; 2) the vibrational distribution nearly obeys the Boltzmann law up to $v=3$, highest vibrational level detected; the vibrational temperature is about $2400 \mathrm{~K}$; 3) the $\mathrm{H}_{2}$ partial pressure is about $70 \%$ of the pressure measured by the gauge, indicating some level of dissociation; 4) the atomic fraction of $\mathrm{H}$ atoms is small $(\sim 5 \%)$, but their apparent translational temperature is high $(2500 \mathrm{~K})$, yielding a high partial pressure (ref. 11).

New measurements have been attempted to assess the inhomogeneities within the reactor and the kinetics of molecular excitation by electrons and relaxation by walls. These are illustrated in Fig. 5 and 6 . Figure 5 shows the rise and fall of rotational temperature in $\mathrm{v}=0$ and of the vibrational temperature (derived from the densities of $\mathrm{v}=0$ and 1 ) under excitation by a rectangular discharge pulse of $10 \mathrm{~A}$ current and $1 \mathrm{~ms}$ duration. Figure 6 presents the radial steady-state distributions of the vibrational and rotational temperatures and their rise under excitation by the pulse leading edge. The difference between them is striking, as the rotational temperature presents a strong gradient between the center and the wall, while the vibrational distribution is flat. This is due to the difference in deactivation rates at the wall.

A preliminary discussion of these phenomena can be found in ref. 20. In summary:

Rotational relaxation is governed by two competing processes: 1) R-T relaxation followed by transport of the waste translational energy to the walls at $\sim$ the sound speed, and 2) diffusion of rotationally hot molecules to the walls with nearly unit probability collisional deactivation at the wall. The second one dominates at low pressure. We estimate that the first one becomes about as fast at $55 \mu \mathrm{bar}$ and rapidly takes over as the pressure increases. These estimates are naturally valid only for our reactor geometry.

Vibrational relaxation is dominated at all pressures by diffusion to the walls followed by collisional deactivation. About seven wall collisions are necessary to deactivate $\mathrm{v}=1$, based on measurements taken at the lower pressure of $5.5 \mu \mathrm{bar}$ to reduce the influence of diffusion.

\section{CONCLUSION}

It is clear that many types of low pressure discharges can be tackled by CARS. New information about the kinetics of plasmas obviously will be obtained. However, some progress remains to be made in order to achieve the detection sensitivities necessary to monitor the high $v$ states, which frequently are the main intermediates to molecular 
dissociation by low energy electrons. We feel the potential exists for a factor of ten improvement in detectable densities if the window stray light problem can be solved. Very little progress was made on this front over the past three years in our laboratory, and this is undoubtedly one of the difficult technical challenges of CARS for tomorrow.

\section{REFERENCES}

1. W.M. Tolles, J.W. Nibler, J.R. McDonald and A.B. Harvey, Appl. Spectry. $\underline{31}, 253$ (1977).

2. M. Pealat, D. Debarre, J.J. Marie, J.P.E. Taran, A. Tramer and C.B. Moore, Chem. Phys. Letters, 98, 299 (1983).

3. D.P. Gerrity and J.J. Valentini, J. Chem. Phys., 81, 1298 (1984).

4. T. Dreier and J. Wolf rum, Appl. Phys., B33, $213(1984)$.

5. D. Debarre, M. Lefebvre, M. Pealat, J.P.E. Taran, D.J. Bamford and C.B. Moore, J. Chem. Phys., 83, 4476 (1985).

6. J.W. Nibler, J.R. McDonald and A.B. Harvey, Opt. Commun., 18, 371 (1976).

7. W.M. Shaub, J.W. Nibler and A.B. Harvey, J. Chem. Phys., 67,1883 (1977).

8. V.V. Smirnov and V.I. Fabelinskii, JETP Letters, 28, 427 (1978).

9. M. Pealat, J.P.E. Taran, J. Taillet, M. Bacal and A.M. Bruneteau, J. Appl. Phys., $\underline{52}$, 2687 (1981).

10. S.I. Valyanskii, A. Vereshchagin, V. Vernke, A. Yu Volkov, P.P. Pashinin, V.V. Smirnov, V.I. Fabelinskii and P.L. Chapovskii, Sov. J. Quant. Elec., 14, 1226 (1984).

11. M. Pealat, J.P.E. Taran, M. Bacal and F. Hillion, J. Chem. Phys., 82, 4943 (1985).

12. S. Druet and J.P.E. Taran, Prog. Quant. Elect., 7, 1 (1981).

13. M. Moisan, C. Baudry and P. Leprince, IEEE Trans.'Plasma Sci., PS3, 55 (1975).

14. B. Massabieaux, G. Gousset, M. Lefebvre and M. Pealat, to be published.

15. C.F. Treanor, J.W. Rich and R.G. Rehm, J. Chem. Phys., 48, 1798 (1968); B. F. Gordiets, Sh. S. Mamedov and L. A. Shelepin, Sov. Phys. JETP, 48, 648 (1974).

16. M. Capitelli, M. Dilonardo and C. Gorse, Chem. Phys., 56, 29 (1981).

17. H. Brunet and J. Rocca-Serra, J. Appl. Phys., 57, 1574 (1985).

18. L.M. Cheung, D.M. Bishop, D.L. Drapcho and G.M. Rosenblatt, Chem. Phys. Lett., 80, 445 (1981); J.L. Hunt, J.D. Poll and L. Wolniewicz, Can. J. Phys., 62, 1719 (1984).

19. M. Pealat, M. Lefebvre, J.P.E. Taran and P.L. Kelley, to be published.

20. M. Lefebvre, M. Pealat, J.P.E. Taran, F. Hillion and M. Bacal "CARS Time Resolved Measurements of $\mathrm{H}_{2}$ Rovibrational Populations in an $\mathrm{H}$ - source", presented at the 2nd European Workshop on the Production of Light Negative Ions, Palaiseau, France, March 5-7 (M. Bacal and C. Moutet, Ed.), Ecole Polytechnique, Palaiseau, France, 1986. 